\title{
Temporal Associations Between Pain-Related Factors and Abnormal Muscle Activities in a Patient with Chronic Low Back Pain: A Cross-Lag Correlation Analysis of a Single Case
}

This article was published in the following Dove Press journal:

Journal of Pain Research

\author{
Hayato Shigetoh (iD) ${ }^{1,2}$ \\ Yuki Nishi ${ }^{1}$ \\ Michihiro Osumi ${ }^{3}$ \\ Shu Morioka (iD) ${ }^{1,3}$ \\ 'Department of Neurorehabilitation, \\ Graduate School of Health Sciences, Kio \\ University, Koryo, Nara, Japan; ${ }^{2}$ Miura \\ Internal Medicine Michiko Pediatrics \\ Clinic, Marugame City, Kagawa, Japan; \\ ${ }^{3}$ Neurorehabilitation Research Center, \\ Kio University, Nara, Japan
}

\begin{abstract}
Purpose: The cross-sectional and longitudinal associations between pain-related factors and muscle activity in patients with chronic low back pain (CLBP) are unclear. This study aimed to examine the temporal associations between them in a CLBP patient using a single-case analysis to account for an individual course.
\end{abstract}

Patient and Methods: A patient with a history of lower back pain lasting more than 3 months was studied from March 16, 2020 to May 30, 2020. Surface electromyographic signals were recorded from over the bilateral lumbar erector spinae in the patient while performing a standing trunk flexion and re-extension task. The average value for muscle activity during each movement phase was estimated, and the flexion relaxation ratio (FRR) of all channels was subsequently calculated. Pain-related factors and disability were assessed using questionnaires. All assessments were performed nine times, along with 2-3 months of intervention. Once or twice per week, the patient received physical therapy that consisted of soft tissue mobilization, joint mobilization, nerve mobilization, and patient education. A cross-lag correlation analysis of this single case was conducted.

Results: Pain-related factors showed a trend toward improvements in all variables when compared to those in the first assessment; however, there was no general change (increase) in FRR over time. The cross-lag correlation analysis revealed that improvements in FRR were associated with improvements in body perception disturbance $(\rho=-0.78, \mathrm{p}<0.01)$, and that improvements in muscle activity during the extension phase were associated with improvements in pain $(\rho=0.75)$, psychological factors $(\rho=0.57)$, and disability $(\rho=0.67)(\mathrm{p}<0.05)$. Conclusion: Our findings suggest that improvements in body perception were temporally associated with improvements in FRR, and improvements in pain, psychological factors, and disability were temporally associated with a reduction in muscle activity during the trunk extension phase in this patient with CLBP.

Keywords: chronic low back pain, pain-related factors, abnormal muscle activities, crosslag correlation

\section{Introduction}

Interventions focusing on disability are essential for patients with low back pain (LBP). ${ }^{1-3}$ LBP-related disability is associated with not only pain severity but also several factors, including psychological, social, and biophysical factors. ${ }^{4}$ In particular, psychological factors are often correlated with disability in individuals with
Correspondence: Hayato Shigetoh

Graduate School of Health Sciences, Kio University,

Koryo, 4 Chome-2-2 Umaminaka, Kitakatsuragi District, Nara 635-0832, Japan

Tel $+8 \mid 74554$ I60I

Fax +81745541600

Email hayato.ptlI2I@gmail.com
Journal of Pain Research 2020:13 3247-3256

3247 
chronic low back pain (CLBP) ${ }^{5-11}$ Recently, body perception disturbance (ie, lumbar proprioceptive deficits, body image disorder, and neglect-like symptoms) has been reported to affect disability in patients with CLBP. ${ }^{12}$ As disability in CLBP can be observed as an abnormal muscle activity, ${ }^{13,14}$ physical therapists should therefore assess muscle activity in patients with CLBP in clinical practice. Impairment in muscle activity is attributable to various pain-related factors, such as psychological factors and body perception disturbance through the central nervous system. ${ }^{15,16}$ However, the cross-sectional and longitudinal associations between pain-related factors and muscle activity in patients with CLBP are unclear.

Erector spinae activity during trunk flexion is reportedly altered in patients with LBP. ${ }^{17}$ The muscle activity in these patients does not relax in the full trunk flexion position ${ }^{18}$ and excessively increases when extending from trunk flexion. ${ }^{19}$ These characteristic muscle activities have been reported to be altered due to pain itself $\mathrm{f}^{20}$ and psychological factors. ${ }^{18,21}$ Nonetheless, temporal changes in the relationships between abnormal muscle activity and pain-related factors remain unclear. Previous case-series have reported that changes in pain-related factors over time vary considerably from case to case ${ }^{22,23}$ therefore, it is advisable to analyze every case.

The present study aimed to examine the temporal associations between pain-related factors and abnormal muscle activities using a single-case analysis to account for an individual course. Identifying these temporal associations could support tailor-made interventions that take painrelated factors that lead to improvements in muscle activity and disability into account. We hypothesized that temporal improvements in pain-related factors occur before temporal improvements in specific muscle activities. Additionally, focusing on the muscle activity during each movement phase, we expected that pain-related factors were associated with excessively increased muscle activity in both the full trunk flexion phase and the trunk extension phase.

\section{Patient and Methods}

\section{Study Setting and Patient Selection \\ Criteria}

This study was a single case experimental design. The study period was from March 16, 2020 to May 30, 2020. The study was conducted at the Miura Internal Medicine Michiko Pediatrics Clinic. Inclusion criteria included LBP lasting more than 3 months. The LBP area was defined as the area bounded by the lowest palpable rib superiorly and the gluteal folds inferiorly. ${ }^{24}$ Exclusion criteria included central nervous system disease, dementia, LBP that appeared within the previous 3 months, difficulty understanding questionnaires and tasks, and difficulty performing tasks.

The study protocol conformed to the Declaration of Helsinki and was approved by the ethics committee of Kio University Health Sciences Graduate School (approval no. H30-06). We explained the study protocol to the patient and obtained written informed consent to publish the case report.

\section{Case Description}

The patient was a 67-year-old man with lumbar spinal stenosis who worked in the trash collection business and often carried heavy loads. The patient felt pain from his lower back to the posterior aspect of both lower extremities and feet. He experienced LBP several times for over 10 years and started to feel pain and numbness at the lower extremities a few months ago. Furthermore, the patient expressed that his pain was aggravated during lifting, standing, and walking, and was eased while sitting and lying.

The clinical findings were as follows. Several nerve tension tests, including the slump test, straight-leg-raising test, and Bragard test, demonstrated bilateral positive signs with more severe symptoms on the right side. During standing forward trunk flexion, neck flexion exacerbated the pain, whereas neck extension reduced the pain, indicating that the pain might be associated with decreased nerve sliding. ${ }^{25}$ The areas with sensory dullness were the posterior thigh surface and lower legs on both sides. Muscle weakness was observed in the tibialis anterior and extensor hallucis longus, particularly on the right side. In the active movement test, trunk flexion and extension were slightly restricted and restricted by pain, respectively. In the joint play test, hypomobility was noted from the upper lumbar spine to the thoracic spine, especially at L3, L4, and L5, with symptoms observed in the right lower extremity. Muscle spasm was detected in the erector spinae, quadratus lumborum, tensor fascia lata, hamstrings, gastrocnemius, and gluteus maximus.

\section{Assessment Time Points}

Assessments in this study were periodically conducted using the recommended number of assessments (a total of 10-16 observations in the data stream) for a single-case time-series analysis as a guide ${ }^{26}$ and were performed at 
7-10-day intervals to coincide with outpatient rehabilitation implementation days.

\section{Evaluations of Patient Characteristics Using Questionnaires}

Pain intensity, body perception disturbance, psychological factors, and disability were assessed in the patient using the Short-Form McGill Pain Questionnaire-2 (SFMPQ-2), Fremantle Back Awareness Questionnaire (FreBAQ), Örebro Musculoskeletal Screening Questionnaire-12 (OMSQ-12), and Patient-Specific Functional Scale (PSFS), respectively.

The SFMPQ-2 was used for pain intensity assessment. This questionnaire consists of 22 pain descriptors that the patient rates on an 11-point numerical rating scale based on pain severity. ${ }^{27}$ The total score is calculated from the sum of the 22 items with a higher score indicating more severe pain. The SFMPQ-2 comprises one affective subscale and three sensory subscales, with the sensory subscales further subdivided into three sensory pain types: continuous pain, intermittent pain, and predominantly neuropathic pain. SFMPQ-2 had good internal consistency (SFMPQ-2-total: Cronbach's $\alpha=0.83$ ) and reliability (intraclass correlation coefficient $[\mathrm{ICC}]=0.907)$. ${ }^{27}$

The FreBAQ was applied to assess the patient's body perception disturbance. This questionnaire comprises nine items and three subscales (proprioception, body image, and neglect-like symptoms) ${ }^{28}$ with a higher score indicating more severe body perception disturbance. The FreBAQ had acceptable internal consistency (Cronbach's $\alpha=0.80)$ and reliability $\left(\operatorname{ICC}_{3,1}=0.81\right){ }^{28}$

The OMSQ-12 contains 12 items $^{29}$ and is a shorter version of the original 21-item OMSQ, which consists of six factors (physical function, psychological, problem, personal, other, and fear avoidance) ${ }^{30}$ For this study, the OMSQ-12 was employed to evaluate psychological factors. Therefore, the following items that correspond to psychological factors were extracted: item 5 ("During the past 2-3 days, rate how tense or anxious you have felt"), item 6 ("During the past 2-3 days, rate how 'depressed' or 'down' you have felt"), and item 7 ("What do you think is the risk that your current pain or problem will not improve?"). We used the total value for items 5, 6, and 7 as psychological factors, with higher scores indicating more severe psychological conditions. The OMSQ-12 had good reliability. The $\operatorname{ICC}_{(2,1)}$ for each OMSQ-12 item ranged from $0.71-0.99$, and was 0.92 for the total
OMSQ-12 score. The OMSQ-12 correlated $(r=0.97)$ with the original 21-item questionnaire. ${ }^{30}$

The PSFS was used to assess personalized disability. ${ }^{31}$ At the initial assessment, the patient listed three activities of daily living. Each item was rated on an 11-point numerical rating scale in response to the question, "How difficult is it for you to perform this activity because of your back pain?" anchored by $0=$ able and $10=$ unable. The most disabling activity for the patient was lifting and carrying heavy loads, with higher scores indicating more severe disability. PSFS had good reliability $(\mathrm{ICC}=0.82) .{ }^{32}$ There was a significant correlation between Global Rating of Change $(r=0.82)$ and Numerical Rating Scale $(r=0.80){ }^{32}$

\section{Standing Trunk Flexion and Re-Extension Task}

The patient was instructed to perform a standing trunk flexion and re-extension task while electromyographic (EMG) signals were recorded. The phases of this task were categorized into standing, flexion, full flexion, and extension phases, with each phase lasting for $3 \mathrm{~s}^{33}$ (Figure 1).

The patient began the task by standing without movement (standing phase) with his feet at hip-width. After the first auditory signal, the patient bent forward with a slow, controlled movement (flexion phase) to reach maximum trunk flexion before the second auditory signal. The patient was subsequently instructed to maintain the full flexion position (full flexion phase) until the third auditory signal. After the third auditory signal, the patient returned to the upright posture for $3 \mathrm{~s}$ (extension phase). After completing a reference trial at least once, the patient repeated the task for three trials. The patient experienced LBP during the performance of the task.

\section{Recording and Analysis of Muscle Activities}

Surface EMG signals were detected using a grid of sheettype electrodes (Unique-Medical Inc., Brooklyn, NY, USA). The EMG grid was comprised of five fourchannel electrodes and had an inter-electrode distance of $25 \mathrm{~mm}$ in both the horizontal and vertical direction. The patient's glabrous skin over the erector spinae was cleaned with alcohol. Two grids of sheet-type electrodes coated with an electroconductive gel were taped to the skin at that site. The electrode was located $3 \mathrm{~cm}$ outside of the midpoint of the lumbar spinous process and covered upward along the erector spinae from the level of the Jacoby line 


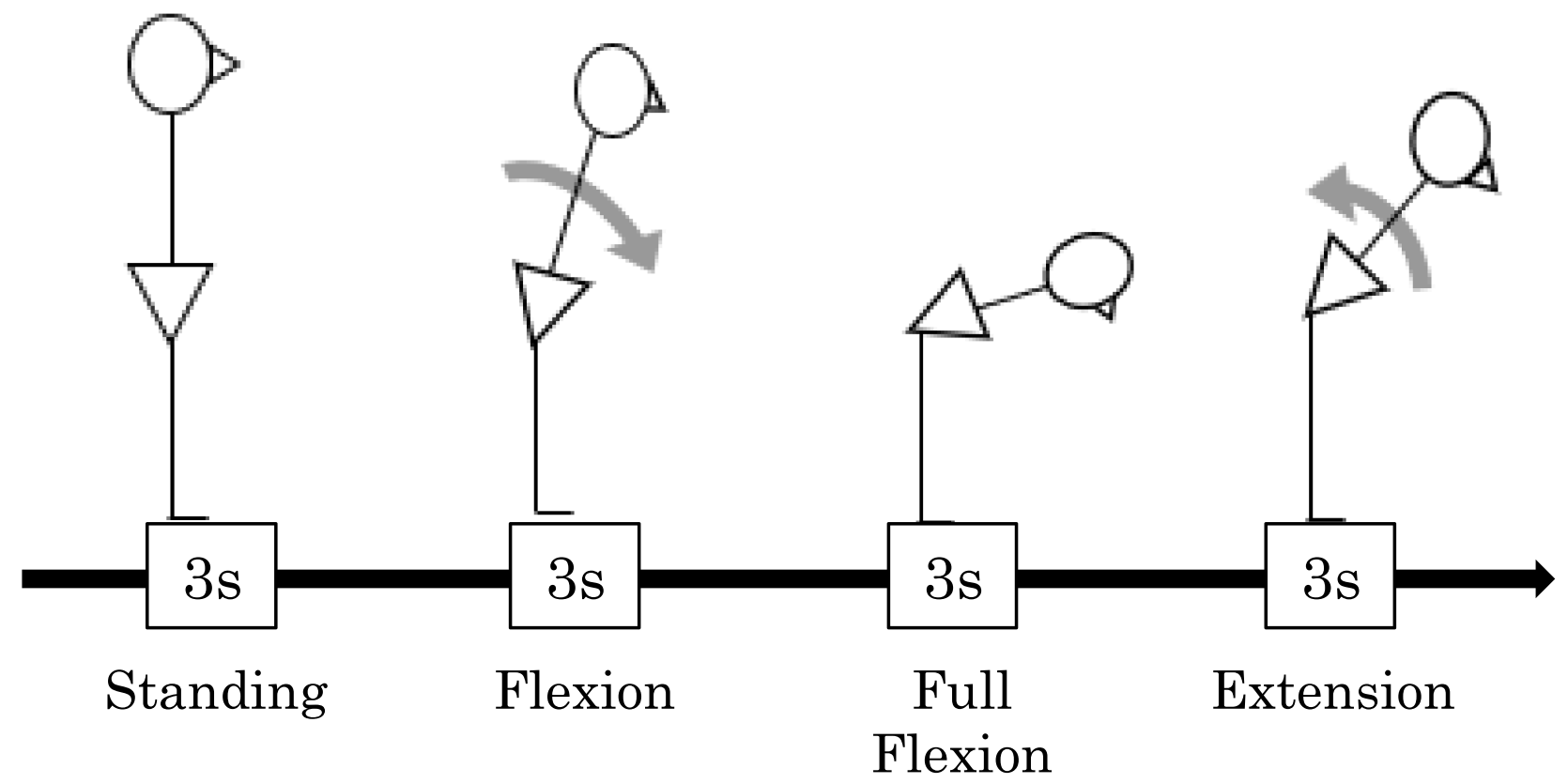

Figure I The standing, trunk flexion and re-extension task.

(Figure 2). A reference electrode was placed over the radial styloid process on the left side. The recorded EMG signals (sampling rate: $1000 \mathrm{~Hz}$ ) were analyzed after bandpass filtering (10 and $400 \mathrm{~Hz}$ ). The root mean square (RMS) EMG values were calculated for each movement phase in both the reference trial and experimental trials. Once the muscle electric signal was captured, it was analyzed, or processed, using the RMS value, which has been widely utilized. In this form of processing, the EMG signal is submitted to mathematical treatments that are designed to quantify the intensity and duration of several events. In other words, RMS measures the muscle activity. Subsequently, normalized RMS EMG values during each phase were calculated by dividing the mean RMS value obtained from the experimental trials by the mean RMS value obtained from the extension phase of the reference

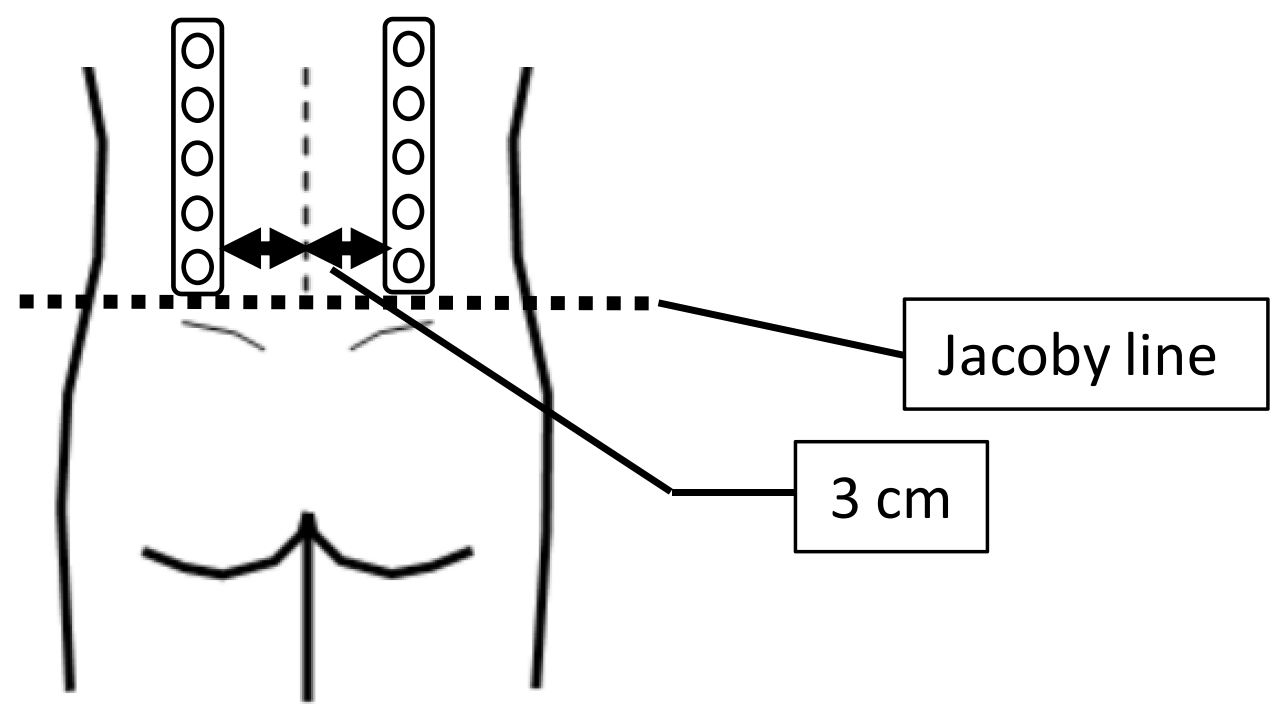

Figure 2 The approximate position of the EMG grid. The EMG electrode grid was placed $3 \mathrm{~cm}$ lateral to the lumbar spinous process on the bilateral erector spinae. 
trial. ${ }^{20}$ Normalized RMS values during the full flexion phase and extension phase were extracted for statistical analysis. If noise could not be removed, RMS values were excluded from the analysis.

For FRR calculation, the maximum surface EMG value during the flexion phase and the mean surface EMG value during the full flexion phase were determined as described. ${ }^{34}$ The FRR of each channel was calculated by dividing the maximum surface EMG value during the flexion phase by the mean surface EMG value during the full flexion phase. ${ }^{34}$ The average FRR of all channels was used as the FRR in the statistical analysis. EMG data were analyzed using a custom-written MATLAB code (v.2019b; MathWorks, Natick, MA, USA).

\section{Interventions}

The patient received physical therapy once or twice per week. This consisted of soft tissue mobilization, joint mobilization, nerve mobilization, and patient education. Soft tissue mobilization, including transverse friction massage and functional massage, was used for pain reduction and muscle relaxation in the area of muscle spasm. Joint mobilization was employed to reduce pain and increase the segmental mobility of the lumbar and thoracic spine. It was performed using a segmental distraction (traction direction was cranial to caudal) with the patient in a side-lying position and a segmental distraction (traction direction was vertical to the facet joint articular surface) with the patient in a prone position. Nerve mobilization was applied to the sciatic nerve to reduce pain and increase nerve sliding. In the side-lying position, the patient was asked to extend the neck while simultaneously extending the lower extremities and dorsiflexing the ankles, followed by flexing the neck while simultaneously flexing the lower extremities. This procedure was repeated 10 times. Patient education included pain management instruction mainly related to biomechanics, such as movement instruction on lifting movement methods and frequent posture changes without holding the same position for long periods. While systematic pain neuroscience education was not provided to the patient, an "overview of pain sensitization" and information regarding how "pain is inherently meant to be a warning signal" were provided.

\section{Statistical Analyses}

FRR and RMS values during the full flexion phase and extension phase were calculated as muscle activity measures. Cross-lag correlation analysis adjusted for autocorrelation was performed using the Simulation Modeling Analysis version 8.3.3. (http://clinicalresearcher.org) to assess the temporal associations between pain-related factors and muscle activity. ${ }^{35}$ This analysis estimates the temporality (lag) of associations between two variables in a single-case experimental design after adjustment for autocorrelation. Correlations between pain-related factors and muscle activity were estimated and compared at lags -2 to +2 . Negative lag denoted a change in pain-related factors before muscle activity (eg, Negative lag (lag $=-1)$ indicated that the muscle activity correlated to pain-related factors of the before 1 assessment time point). Positive lag denoted a change in pain-related factors after muscle activity (eg, Positive lag $(\operatorname{lag}=+1)$ indicated that the muscle activity correlated to pain-related factors of the after 1 assessment time point). A zero lag indicated that pain-related factors changed contemporaneously with muscle activity. The number of simulations was set at 10,000 . The significance level was set at $p<0.05$.

\section{Results}

\section{Patient Characteristics}

The progression of all outcomes over time is presented in Table 1. Measurements were performed nine times before the patient was unable to come to rehabilitation due to a work conflict. Pain-related factors showed a trend toward improvements in all variables when compared to those in the first assessment, with repeated worsening and improvement. There was no obvious trend across measurement time points for muscle activity.

\section{Cross-Lag Correlation Analysis}

Cross-lag correlation analysis revealed that most painrelated factors changed concomitantly with muscle activity, with the strongest correlations observed at zero lag. Hence, the present study focused on cross-lag correlation analysis at zero lag according to a previous study. ${ }^{23}$

\section{Cross-Lag Correlation Analysis Between Muscle Activity and Pain-Related Factors}

The correlation matrices between each variable at zero lag are shown in Table 2. The correlation coefficients between each of the pain-related factors and muscle activity at zero lag are shown in Figure 3. FRR was significantly negatively correlated with FreBAQ at zero lag. No significant correlation between muscle activity in the full flexion 
Table I Patient Characteristics

\begin{tabular}{|c|c|c|c|c|c|c|c|c|c|}
\hline & \multicolumn{9}{|c|}{ Number of Observations } \\
\hline & lst & 2nd & 3rd & 4th & 5th & 6th & 7th & 8th & 9th \\
\hline SFMPQ-2-total & 71 & 31 & 35 & 32 & 29 & 32 & 16 & 20 & 28 \\
\hline FreBAQ & 28 & 12 & 5 & 11 & 7 & 6 & 0 & 1 & 5 \\
\hline OMSQ-12-psychological factor & 26 & 12 & 15 & 8 & 11 & 11 & 10 & 12 & 13 \\
\hline PSFS & 8 & 4 & 6 & 6 & 4 & 5 & 4 & 6 & 6 \\
\hline FRR & 4.11 & 3.51 & 4.52 & 3.78 & 3.99 & 3.58 & 4.85 & 4.67 & 4.49 \\
\hline RMS (full flexion phase) & 0.93 & 0.91 & 0.96 & 0.94 & 0.92 & 0.95 & 0.83 & 0.89 & 0.95 \\
\hline RMS (extension phase) & 1.01 & 0.97 & 1.02 & 1.01 & 1.00 & 1.00 & 0.91 & 0.96 & 1.01 \\
\hline
\end{tabular}

Abbreviations: SFMPQ-2, Short-Form McGill Pain Questionnaire-2; PSFS, Patient-Specific Functional Scale; FreBAQ, Fremantle Back Awareness Questionnaire; OMSQ-I2, Örebro Musculoskeletal Screening Questionnaire-12; FRR, flexion relaxation ratio; RMS, root mean square.

Table 2 Cross-Correlations at Zero Lag Indicating the Level of Cross-Sectional Associations Over Time Between Pain-Related Factors and Muscle Activity

\begin{tabular}{|l|l|l|l|l|l|l|l|}
\hline & $\begin{array}{l}\text { SFMPQ- } \\
\text { 2-Total }\end{array}$ & FreBAQ & $\begin{array}{l}\text { OMSQ-12- } \\
\text { Psychological Factor }\end{array}$ & PSFS & FRR & $\begin{array}{l}\text { RMS (Full } \\
\text { Flexion Phase) }\end{array}$ & $\begin{array}{l}\text { RMS } \\
\text { (Extension } \\
\text { Phase) }\end{array}$ \\
\hline $\begin{array}{l}\text { SFMPQ-2-total } \\
\text { FreBAQ }\end{array}$ & $\mathrm{I}$ & & & & & & \\
OMSQ-12-psychological & $0.70^{*}$ & $\mathrm{I}$ & & & \\
factor & 0.42 & 0.19 & $\mathrm{I}$ & & & \\
PSFS & $0.5 \mathrm{I}$ & 0.18 & 0.57 & 1 & & \\
FRR & -0.48 & $-0.78^{* *}$ & 0.16 & 0.21 & 1 & \\
RMS (full flexion phase) & 0.44 & 0.16 & 0.37 & 0.47 & -0.22 & 1 \\
RMS (extension phase) & $0.75^{*}$ & 0.34 & $0.57^{*}$ & $0.67^{*}$ & -0.13 & $0.91 * *$ \\
\hline
\end{tabular}

Note: $*_{p}<0.05, * * p<0.01$.

Abbreviations: SFMPQ-2, Short-Form McGill Pain Questionnaire-2; PSFS, Patient-Specific Functional Scale; FreBAQ, Fremantle Back Awareness Questionnaire; OMSQ-12, Örebro Musculoskeletal Screening Questionnaire-12; FRR, flexion relaxation ratio; RMS, root mean square.

phase and pain-related factors was observed at zero lag. However, muscle activity in the extension phase showed significant positive correlations with SFMPQ-2-total, OMSQ-12-psychological factor, and PSFS.

\section{Discussion}

The present study investigated the temporal associations between pain-related factors and abnormal muscle activity in a patient with CLBP via a cross-lag correlation analysis of a single case. Cross-lag correlation analysis revealed that improvements in most pain-related factors (pain intensity, body perception disturbance, psychological factors, and disability) were most strongly associated with improvements in specific muscle activity at zero lag. In other words, these findings suggested that pain-related factors and specific muscle activity simultaneously improved. Furthermore, improvements in FRR were associated with improvements in body perception disturbance, and muscle activity in the full trunk flexion phase was not temporally related to pain-related factors. However, improvements in specific muscle activity during the extension phase were associated with improvements in pain intensity, psychological factors, and disability. The present study is the first to show how pain-related factors are temporally associated with specific muscle activity in a patient with CLBP.

Concerning muscle activity during the full trunk flexion phase, improvements in RMS were not associated with improvements in pain-related factors; nevertheless, improvements in FRR were related to improvements in body perception disturbance. This result suggested that body perception disturbance might make the muscle stiff during the full trunk flexion phase. Back muscle stiffness has been reported to be associated with disability in patients with $\mathrm{LBP}^{36}$ A previous cross-sectional study showed that the reduction in FRR was not associated with pain intensity. ${ }^{37}$ The present study obtained similar results and showed that improvements in FRR were not related to improvements in pain intensity. 


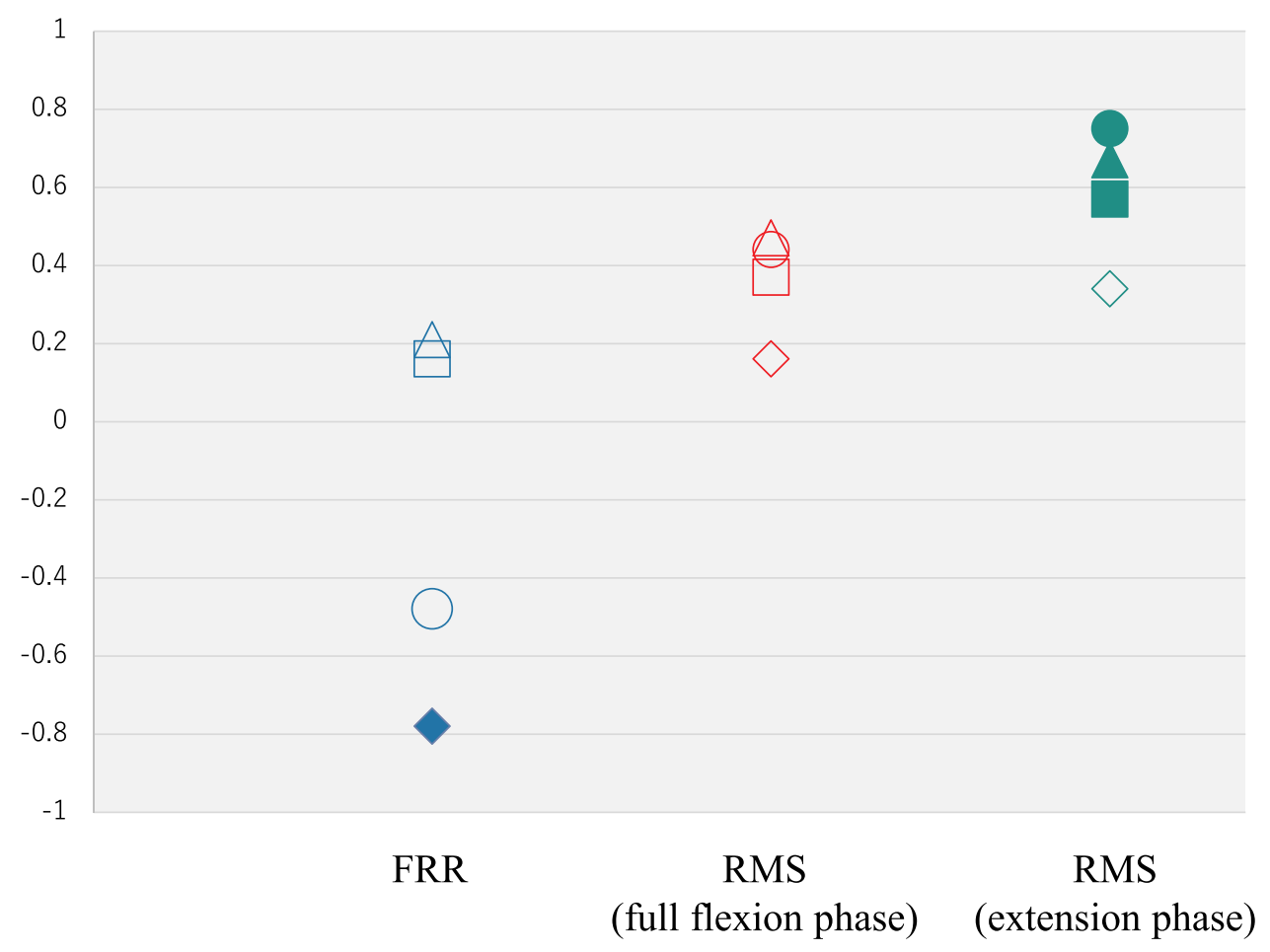

Figure 3 The correlation coefficients between each of the pain-related factors and muscle activity at zero lag.

Notes: Color-filled markers: indicate correlations that reached statistical significance. Unfilled markers: indicate correlations that not reached statistical significance. Circle markers: indicate correlations between the SFMPQ-2 and muscle activity. Triangle markers: indicate correlations between the PSFS and muscle activity. Square markers: indicate correlations between the OMSQ-12-psychological factor and muscle activity. Rhombus markers: indicate correlations between the FreBAQ and muscle activity. Abbreviations: SFMPQ-2, Short-Form McGill Pain Questionnaire-2; PSFS, Patient-Specific Functional Scale; FreBAQ, Fremantle Back Awareness Questionnaire; OMSQ- 2, Örebro Musculoskeletal Screening Questionnaire-12; FRR, flexion relaxation ratio; RMS, root mean square.

Another previous study reported that patients with decreased proprioception may have increased antagonist muscle activity. ${ }^{38}$ In the present case, improvements in FRR were associated with improvements in body perception disturbance; furthermore, improvements in muscle activity during the extension phase were not related to improvements in body perception disturbance, which might be attributable to the difference between agonist and antagonistic muscle activities in the movement phase. Body perception changes have been reported to result in increased or decreased muscle activity. ${ }^{39,40}$ Additionally, it has been suggested that body perception changes induce unconscious motor adaptations that require changes in muscle activity. A comprehensive model showing the influence of sensorimotor incongruence on muscle activity through the central nervous system has been proposed. ${ }^{16}$ Hence, in the present study, the improvements in body perception disturbance might be related to the improvements in FRR resulting from unconscious motor adaptation through the central nervous system. Therefore, body perception might be an essential factor for the reduction in excessive muscle stiffness in the present case.
In the present study, improvements in muscle activity during the extension phase were associated with improvements in pain intensity, psychological factors, and disability. Previous studies reported increased or decreased spinal erector muscle activity in patients with LBP when extending from forward trunk flexion. ${ }^{19,20}$ Although the relationship between muscle activity and pain remains controversial, improvements in pain were associated with improvements in muscle activity during the extension phase in the patient described in the present study. A previous study reported that exacerbation of psychological factors leads to increased muscle activity. ${ }^{41}$ A comprehensive model in which pain, psychological factors, and body perception disturbance comprehensively affect muscle activity through the central nervous system has been proposed. ${ }^{16}$ Thus, improvements in nociceptive stress and psychological factors modified pain and muscle activity consequent to comprehensive motor adaptations, and consequently modified disability. These might have been temporally related to improvements in muscle activity during the extension phase and might have further led to a temporal association between improvements in muscle 
activity during the extension phase and improvements in disability.

The present study suggested that each pain-related factor results in muscle activity adaptations during each movement phase. Concerning treatment, the effectiveness of cognitive functional therapy $y^{23,42}$ and graded sensorimotor re-education ${ }^{22}$ as interventions for psychological factors and body perception disturbance in patients with CLBP has been reported. Considering the temporal influence of pain-related factors on muscle activity, the abovementioned interventions should be combined with a motor control approach for specific muscle activity in patients with CLBP.

The present study has several limitations. First, as this study had a single-case design, the results captured individual characteristics; hence, the generalizability of our findings to all patients with CLBP is limited. Second, the measurements in this study were performed nine times, which does not meet the recommended number of measurements for a single-case time-series analysis. ${ }^{26}$ Nonetheless, we believe that actual measurements of variables can capture temporal associations between variables over time, and the correlation coefficients can be used as a reference for trends in temporal associations between variables. Third, the standing trunk flexion and reextension task performed by the patient was based on a previous study; although the time was set during each movement phase, the velocity of the patient's movements could not be adequately controlled. For this reason, individual differences in fine velocity might have influenced muscle activity. Fourth, pain intensity outcomes were assessed using questionnaires, which might have resulted in subjective bias. Fifth, we did not assess task-specific movement fear or psychological stress to work. Therefore, the evidence in this case was insufficient to assess the effect of task-specific psychological factors and psychological stress to work on muscle activity.

\section{Conclusion}

To our knowledge, this is the first study to investigate the temporal associations between pain-related factors and muscle activity in a single patient with CLBP. Our findings suggest that improvements in body perception in a patient with CLBP are temporally associated with improvements in FRR; and that improvements in pain, psychological factors, and disability in a patient with CLBP are temporally associated with the reduction of muscle activity during the trunk extension phase. Our findings could aid in the development of a longitudinal approach that considers pain-related factors, leading to improved motor control.

\section{Acknowledgments}

We would like to express our gratitude to the patient and clinical staff involved in the study described in the manuscript.

\section{Disclosure}

The authors report no conflicts of interest in this work.

\section{References}

1. Hoy D, Bain C, Williams G, et al. A systematic review of the global prevalence of low back pain. Arthritis Rheum. 2012;64 (6):2028-2037. doi:10.1002/art.34347

2. Kamper SJ, Henschke N, Hestbaek L, Dunn KM, Williams CM. Musculoskeletal pain in children and adolescents. Brazilian $J$ Phys Ther. 2016;20(3):275-284. doi:10.1590/bjpt-rbf.2014.0149

3. Hartvigsen J, Christensen K, Frederiksen H. Back pain remains a common symptom in old age. A population-based study of 4486 Danish twins aged 70-102. Eur Spine J. 2003;12(5):528-534. doi:10.1007/s00586-003-0542-y

4. Hartvigsen J, Hancock MJ, Kongsted A, et al. What low back pain is and why we need to pay attention. Lancet. 2018;391 (10137):2356-2367. doi:10.1016/S0140-6736(18)30480-X

5. Shanbehzadeh S, Salavati M, Tavahomi M, Khatibi A, Talebian S, Khademi-Kalantari K. Reliability and validity of the pain anxiety symptom scale in Persian speaking chronic low back pain patients. Spine (Phila Pa 1976). 2017;42(21):E1238-E1244. doi:10.1097/ BRS.0000000000002149

6. Marshall PWM, Schabrun S, Knox MF. Physical activity and the mediating effect of fear, depression, anxiety, and catastrophizing on pain related disability in people with chronic low back pain. PLoS One. 2017;12(7):1-15. doi:10.1371/journal.pone.0180788

7. Chou R, Shekelle P. Will this patient develop persistent disabling low back pain? JAMA. 2010;303(13):1295-1302. doi:10.1001/jama. 2010.344

8. Pinheiro MB, Ferreira ML, Refshauge K, et al. Symptoms of depression as a prognostic factor for low back pain: a systematic review. Spine J. 2016;16(1):105-116. doi:10.1016/j.spinee.2015.10.037

9. Wertli MM, Rasmussen-Barr E, Weiser S, Bachmann LM, Brunner F. The role of fear avoidance beliefs as a prognostic factor for outcome in patients with nonspecific low back pain: a systematic review. Spine J. 2014;14(5):816-836.e4. doi:10.1016/j.spinee.2013.09.036

10. Wertli MM, Eugster R, Held U, Steurer J, Kofmehl R, Weiser S. Catastrophizing - a prognostic factor for outcome in patients with low back pain: a systematic review. Spine J. 2014;14(11):2639-2657. doi:10.1016/j.spinee.2014.03.003

11. Ferrari S, Chiarotto A, Pellizzer M, Vanti C, Monticone M. Pain self-efficacy and fear of movement are similarly associated with pain intensity and disability in Italian patients with chronic low back pain. Pain Pract. 2016;16(8):1040-1047. doi:10.1111/papr.12397

12. Wand BM, James M, Abbaszadeh S, et al. Assessing self-perception in patients with chronic low back pain: development of a back-specific body-perception questionnaire. J Back Musculoskelet Rehabil. 2014;27(4):463-473. doi:10.3233/BMR-140467

13. Dubois JD, Abboud J, St-Pierre C, Piché M, Descarreaux M. Neuromuscular adaptations predict functional disability independently of clinical pain and psychological factors in patients with chronic non-specific low back pain. J Electromyogr Kinesiol. 2014;24(4):550-557. doi:10.1016/j.jelekin.2014.04.012 
14. Xia T, Long CR, Vining RD, et al. Association of lumbar spine stiffness and flexion-relaxation phenomenon with patient-reported outcomes in adults with chronic low back pain - a single-arm clinical trial investigating the effects of thrust spinal manipulation. BMC Complement Altern Med. 2017;17(1):1-15. doi:10.1186/s12906-017-1821-1

15. Butera KA, Fox EJ, George SZ. Toward a transformed understanding: from pain and movement to pain with movement. Phys Ther. 2016;96(10):1503-1507. doi:10.2522/ptj.20160211

16. Hodges PW, Smeets RJ. Interaction between pain, movement, and physical activity: short-term benefits, long-term consequences, and targets for treatment. Clin J Pain. 2015;31(2):97-107. doi:10.1097/ AJP.0000000000000098

17. Hodges PW, Danneels L. Changes in structure and function of the back muscles in low back pain: different time points, observations, and mechanisms. J Orthop Sports Phys Ther. 2019;49(6):464-476. doi:10.2519/jospt.2019.8827

18. Watson PJ, Booker CK, Main CJ, Chen ACN. Surface electromyography in the identification of chronic low back pain patients: the development of the flexion relaxation ratio. Clin Biomech. 1997;12 (3):165-171. doi:10.1016/S0268-0033(97)00065-X

19. Zedka M, Prochazka A, Knight B, Gillard D, Gauthier M. Voluntary and reflex control of human back muscles during induced pain. $J$ Physiol. 1999;520(2):591-604. doi:10.1111/j.1469-7793.1999. 00591.x

20. Dubois J, Piché M, Cantin V, Descarreaux M. Effect of experimental low back pain on neuromuscular control of the trunk in healthy volunteers and patients with chronic low back pain. $J$ Electromyogr Kinesiol. 2011;21(5):774-781. doi:10.1016/j.jelekin.2011.05.004

21. Geisser ME, Haig AJ, Wallbom AS, Wiggert EA. Pain-related fear, lumbar flexion, and dynamic EMG among persons with chronic musculoskeletal low back pain. Clin J Pain. 2004;20(2):61-69. doi:10.1097/00002508-200403000-00001

22. Wand BM, O'Connell NE, Di Pietro F, Bulsara M. Managing chronic nonspecific low back pain with a sensorimotor retraining approach: exploratory multiple-baseline study of 3 participants. Phys Ther. 2011;91(4):535-546. doi:10.2522/ptj.20100150

23. Caneiro JP, Smith A, Linton SJ, Moseley LG, O’Sullivan P. How does change unfold? An evaluation of the process of change in four people with chronic low back pain and high pain-related fear managed with Cognitive Functional Therapy: a replicated single-case experimental design study. Behav Res Ther. 2019;117:28-39. doi:10.1016/j.brat.2019.02.007

24. Hoy D, March L, Brooks P, et al. The global burden of low back pain: estimates from the Global Burden of Disease 2010 study. Ann Rheum Dis. 2014;73(6):968-974. doi:10.1136/annrheumdis-2013-204428

25. Majlesi J, Togay H, Ünalan H, Toprak S. The sensitivity and specificity of the slump and the straight leg raising tests in patients with lumbar disc herniation. J Clin Rheumatol. 2008;14(2):87-91. doi:10.1097/RHU.0b013e31816b2f99

26. Nash MR, Borckardt JJ, Abbasa A, Gray E. How to conduct and statistically analyze case-based time series studies, one patient at a time. J Exp Psychopathol. 2011;2(2):139-169. doi:10.5127/ jep. 012210

27. Maruo T, Nakae A, Maeda L, et al. Validity, reliability, and assessment sensitivity of the Japanese version of the short-form McGill pain questionnaire 2 in Japanese patients with neuropathic and non-neuropathic pain. Pain Med (United States). 2014;15 (11):1930-1937. doi:10.1111/pme.12468
28. Nishigami T, Mibu A, Tanaka K, et al. Validation of the Japanese version of the Fremantle Back Awareness Questionnaire in patients with low back pain. Pain Pract. 2018;18(2):170-179. doi:10.1111/ papr.12586/epdf

29. Takasaki H, Gabel CP. Cross-cultural adaptation of the 12-item Örebro musculoskeletal screening questionnaire to japanese (ÖMSQ-12-J), reliability and clinicians' impressions for practicality. J Phys Ther Sci. 2017;29(8):1409-1415. doi:10.1589/jpts.29.1409

30. Gabel CP, Burkett B, Melloh M. The shortened Örebro musculoskeletal screening questionnaire: evaluation in a work-injured population. Man Ther. 2013;18(5):378-385. doi:10.1016/j.math.2013.01.002

31. Beurskens AJ, De Vet HC, Koke AJ, et al. A patient-specific approach for measuring functional status in low back pain. J Manipulative Physiol Ther. 1999;22(3):144-148. doi:10.1016/ S0161-4754(99)70127-2

32. Cleland JA, Fritz JM, Whitman JM, Heath R. Predictors of short-term outcome in people with a clinical diagnosis of cervical radiculopathy. Phys Ther. 2007;87(12):1619-1632. doi:10.2522/ptj.20060287

33. Massé-Alarie H, Beaulieu LD, Preuss R, Schneider C. Influence of chronic low back pain and fear of movement on the activation of the transversely oriented abdominal muscles during forward bending. J Electromyogr Kinesiol. 2016;27:87-94. doi:10.1016/j. jelekin.2016.02.004

34. Neblett R, Brede E, Mayer TG, Gatchel RJ. What is the best surface EMG measure of lumbar flexion-relaxation for distinguishing chronic low back pain patients from pain-free controls? Clin J Pain. 2013;29 (4):334-340. doi:10.1097/AJP.0b013e318267252d.WHAT

35. Borckardt JJ, Nash MR, Murphy MD, Moore M, Shaw D, O’Neil P. Clinical practice as natural laboratory for psychotherapy research: a guide to case-based time-series analysis. Am Psychol. 2008;63 (2):77-95. doi:10.1037/0003-066X.63.2.77

36. Sions JM, Hicks GE. Back stiffness is associated with physical health and low back pain-related disability in community-dwelling older adults. Pain Med. 2017;18(5):866-870. doi:10.1093/pm/pnw107

37. Alschuler KN, Neblett R, Wiggert E, Haig AJ, Geisser ME. Flexionrelaxation and clinical features associated with chronic low back pain: a comparison of different methods of quantifying flexion-relaxation. Clin J Pain. 2009;25(9):760-766. doi:10.1097/AJP.0b013e3181b56db6

38. Suarez T, Laudani L, Giombini A, et al. Comparison in joint position sense and muscle coactivation between ACL deficient and healthy individuals. 2014.

39. Bourdin P, Martini M, Sanchez-Vives MV. Altered visual feedback from an embodied avatar unconsciously influences movement amplitude and muscle activity. Sci Rep. 2019;9(1):1-9. doi:10.1038/ s41598-019-56034-5

40. Osumi M, Nobusako S, Zama T, Taniguchi M, Shimada S, Morioka S. Sensorimotor incongruence alters limb perception and movement. Hum Mov Sci. 2018;57:(September):251-257. doi:10. 1016/j.humov.2017.09.003

41. Vlaeyen JWS, Seelen HAM, Peters M, et al. Fear of movement/(re) injury and muscular reactivity in chronic low back pain patients: an experimental investigation. Pain. 1999;82(3):297-304. doi:10.1016/ S0304-3959(99)00054-8

42. Cañeiro JP, Ng L, Burnett A, Campbell A, O’Sullivan P. Cognitive functional therapy for the management of low back pain in an adolescent male rower: a case report. J Orthop Sports Phys Ther. 2013;43(8):542-554. doi:10.2519/jospt.2013.4699 


\section{Publish your work in this journal}

The Journal of Pain Research is an international, peer reviewed, open access, online journal that welcomes laboratory and clinical findings in the fields of pain research and the prevention and management of pain. Original research, reviews, symposium reports, hypothesis formation and commentaries are all considered for publication. The manuscript management system is completely online and includes a very quick and fair peer-review system, which is all easy to use. Visit http:// www.dovepress.com/testimonials.php to read real quotes from published authors.

Submit your manuscript here: https://www.dovepress.com/journal-of-pain-research-journal 